\title{
Classical frustration and quantum disorder in spin-orbital models
}

\author{
Jan Zaanen ${ }^{\mathrm{a}, *}$, Louis Felix Feiner ${ }^{\mathrm{b}, \mathrm{c}}$, Andrzej M. Oleś ${ }^{\mathrm{d}, \mathrm{e}}$ \\ a Lorentz Institute for Theoretical Physics, Leiden University, P.O.B. 9506, NL-2300 RA, Leiden, The Netherlands \\ ${ }^{\mathrm{b}}$ Institute for Theoretical Physics, Utrecht University, Princetonplein 5, NL-3584 CC, Utrecht, The Netherlands \\ ${ }^{\mathrm{c}}$ Philips Research Laboratories, Prof. Holstlaan 4, NL-5G5C AA, Eindhoven, The Netherlands \\ d Institute of Physics, Jagellonian University, Reymonta 4, PL-30059, Kraków, Poland \\ e Max-Planck-Institut für Festkörperforschung, Heisenbergstrasse 1, D-70569, Stuttgart, Germany
}

\begin{abstract}
Recently much attention is paid to the role of the orbital degrees of freedom in transition metal oxides as it remains unclear whether they can remain in a quantum disordered state at zero temperature. Discrete symmetry of the orbital sector counteracts the quantum melting, but especially in doped systems there are signs of dynamical frustration involving the spin-, charge-, and orbital sector simultaneously. It was discovered that even the simple Kugel-Khomskii (KK) model, describing $e_{\mathrm{g}}$ degenerate Mott-insulators, is characterized by a point of perfect dynamical frustration on the classical level, reached in the absence of Hund's rule and electron-phonon couplings. This frustration is lifted on the quantum level, and the true nature of the ground state is still unknown. At present there are two proposals: the $\mathrm{KCuF}_{3}$ phase, stabilized by an order-out-of-disorder mechanism; or spin orbital valence bond phases. It will be argued that at least in the Cu-based systems of this kind, the electron-phonon coupling is primarily responsible for driving the systems away from the special point in the phase diagram. C 1999 Elsevier Science S.A. All rights reserved.
\end{abstract}

Keywords: Orbital sector; Hund's rule; Phase diagram

\section{Introduction}

The paradigm of correlated electron physics is based on the idea that for a certain category of systems one better starts out with the electronic structure of the atoms, treating the delocalization of the electrons in the solid as a perturbation. Any student of physics has to struggle through the theory of atomic multiplets, which is rather complicated because of the intricacies associated with orbital angular momentum. At first sight it is therefore remarkable that these orbital degrees of freedom are completely neglected in the main stream of correlated electron physics. Recently the interest in 'orbitals' has been reviving, especially since they appear to be relevant in one way or another in the colossal magnetoresistance (CMR) manganites. In the wake of this development, questions are asked on the relevancy

\footnotetext{
* Corresponding author. Tel.: +31-71-5275506; fax: +31-715275511.

E-mail address: jan@lorentz.leidenuniv.nl (J. Zaanen)
}

of these orbitals in the context of seemingly settled problems like the metal-insulator transition in $\mathrm{V}_{2} \mathrm{O}_{3}$ [1]. In this contribution the authors will review yet another recent development. Even in the Mott-insulating limit, where the physics simplifies considerably, the interplay of orbital and spin degrees of freedom poses a problem of principle.

There are two limits where the role of orbital degeneracy is well understood: (i) the 'band structure limit', which is based on the assertion that electron conditions can be neglected. In any modern local density approximation (LDA) band structure calculation, orbitals are fully taken into account on the one particle level, in so far as the atomic limit is of any relevance. These translate into various bands, giving rise to multisheeted fermi surfaces, etcetera. (ii) The localized, orbital and spin ordered case which will be refered to as the 'classical limit'. In Mott-insulators, orbital degrees of freedom acquire a separate existence in much the same way as the spins of the electrons do. The orbitals can be parametrized by pseudospins and these form together with the physical spins a low energy sector 
which is described by generalizations of the Heisenber, spin-Hamiltonian [2]. These are the spin-orbital models, like the Kugel-Khomskii (KK) model for $e_{\mathrm{g}}$ degenerate cubic cuprates [3]. The 'classically' ordered states. becoming exact in the limit of infinite dimensions $(d \rightarrow \infty)$ and/or large (pseudo) spin $(S \rightarrow \infty)$, define what is usually, meant with orbital and spin order.

The question arises if there are yet other possibilities. The authors started to study this problem quite some time ago [4], well before the subject revived due to the manganities. The motivation was actually related to a theoretical development flourishing in the 1980s: large $N$ theories [5]. By enlarging the symmetry, say from $S U(2)$ to $S U(\mathrm{~N})$ with $N$ large, new saddle points (ordered states) appeal which correspond to the fluctuation dominated (non-perturbative) limit of the large $S /$ large $d$ theories. For a single correlated impurity, orbital degeneracy leads in a natural way to these large $N$ notions. The question was asked if these large $N$ notions could become of relevance in lattice problems. The authors focused on the simple problem of the e.g. Jahn-Teller degenerate Mott-insulator, rediscovering the KK Hamiltonian [3]. The authors tried to tackle this problem using the techniques invented by Arovas and Auerbach for the $S U(\mathrm{~N})$ symmetric Heisenberg model [6]. It was found that the $S U(4)$ symmetry is so badly broken that the large $N$ techniques were of little help, which is another way of saying that the physics of the KK model is not controlled by large global symmetry. However an special approximate solution was found which revealed that the quantum fluctuations are actually enhanced, and this provided motivation to study these fluctuations in more detail starting from the large $S$ limit. In this process it was discovered that the enhancement of the fluctuations is due to the control exerted by a point in parameter space which can be either called an infinite order quantum critical point, or a point of perfect dynamical frustration in the classical limit [7].

This phenomenon will be discussed in the next section. It poses a rather interesting theoretical problem. So much is clear that the ground state clear that the classical limit is lifted by quantum fluctuations and the question is on the character of the true ground state. As will be discussed, either the classical spin orbital older might survive, stabilized by an order-outof-disorder mechanism, or quantum-incompressible valence bond like states might emerge. In Section 3 the role of electron-phonon coupling will be addressed, emphasizing the rather counter-intuitive result of LDA + U electronic structure calculations that phonons play a rather secondary role despite the fact that the lattice deformations are large. Finally, the situation in the manganites will be shortly discussed in Section 4.

\section{The Kugel-Khomskii model and dynamical frustration}

Consider a Mott-insulator which is characterized by orbital degeneracy, besides the usual spin degeneracy. Different from pure spin problems, these spin-orbital problems are rather ungeneric and depend on the precise system under consideration. A simple problem is a cubic lattice of $3 d$-ions in $d^{9}$ configuration: the $\mathrm{KK}$ problem, which directly applies to $\mathrm{Cu}$ perovskites like $\mathrm{KCuF}_{3}$ or $\mathrm{K}_{2} \mathrm{CuF}_{3}$ [3]. The large Mott gap in the charge excitation spectrum simplifies matters considerably and one derives an effective Hamiltonian by insisting on one hole per unit cell, deriving superexchange-like couplings between the spin and orbital degrees of freedom by integrating out virtual charge fluctuations.

The spins are described as usually in terms of an $s u(2)$ algebra $\left(\vec{S}_{i}\right)$. The orbital degrees of freedom are the $e_{\mathrm{g}}$ cubic harmonics $x^{2}-y^{2} \sim|x\rangle$ and $3 z^{2}-r^{2} \sim$ $|z\rangle$, which can be parametrized in terms of pseudospins as $|x\rangle=\left(\begin{array}{l}1 \\ 0\end{array}\right), \quad|z\rangle=\left(\begin{array}{l}0 \\ 1\end{array}\right)$. Pauli matrices $\sigma^{u}(u=x, y, z)$ are introduced acting on these states. Different from the spins, the $S U(2)$ symmetry associated with the pseudospins is badly broken because the orbitals communicate with the underlying lattice. Although the $e_{\mathrm{g}}$ states are degenerate on a single site, this degeneracy is broken by the virtual charge fluctuations, which take place along the interatomic bonds, i.e. in a definite direction with respect to the orientation of the orbitals. It is therefore convenient to introduce operators which correspond to orbitals directed either along or perpendicular to the three cubic axes $\alpha=a, b, c$ given by $\left(\tau_{j}^{\alpha}-\frac{1}{2}\right)$ and $\left(\tau_{j}^{\alpha}-\frac{1}{2}\right)$, where:

$\tau_{i}^{a(b)}=\frac{1}{4}\left(-\sigma_{i}^{z} \pm \sqrt{3 \sigma_{i}^{x}}\right), \quad \tau_{i}^{c}=\frac{1}{2} \sigma_{i}^{z}$.

In terms of these operators, the KK Hamiltonian can be written as $\left(J=t^{2} / U\right.$ and $t$ is the hopping along tile $c$-axis) [7]:

$$
\begin{aligned}
& H_{1}=J \sum_{\langle i j\rangle, \alpha}\left[4\left(\vec{S}_{i} \cdot \vec{S}_{j}\right)\left(\tau_{i}^{\alpha}-\frac{1}{2}\right)\left(\tau_{j}^{\alpha}-\frac{1}{2}\right)\right. \\
& \left.+\left(\tau_{i}^{\alpha}+\frac{1}{2}\right)\left(\tau_{j}^{\alpha}+\frac{1}{2}\right)-1\right] .
\end{aligned}
$$

neglecting the Hund's rule splittings $\propto J_{H}$ of the intermediate $d^{8}$ states $\left(J_{H}\right.$ is the singlet-triplet splitting). Including those up to order $\eta=J_{H} / U$ yields in addition:

$$
\begin{aligned}
& H_{2}=J \eta \sum_{\langle i j\rangle, \alpha}\left[\left(\vec{S}_{i} \cdot \vec{S}_{j}\right)\left(\tau_{i}^{\alpha}+\tau_{j}^{\alpha}-1\right)\right. \\
& \left.+\frac{1}{2}\left(\tau_{i}^{\alpha}-\frac{1}{2}\right)\left(\tau_{j}^{\alpha}-\frac{1}{2}\right)+\frac{3}{2}\left(\tau_{i}^{\alpha} \tau_{j}^{\alpha}-\frac{1}{4}\right)\right] .
\end{aligned}
$$


Eqs. (2) and (3) are rather unfamiliar: they describe a regular Heisenberg spin problem coupled into a Pottslike orbital problem (choose two out of three possibilities $\sim x^{2}-y^{2}, \sim y^{2}-z^{2}, \sim z^{2}-x^{2}$ ).

The oddity of Eqs. (2) and (3) becomes clear when one studies the classical limit. As usually, the $\vec{S}$ s and the $\vec{\tau} \mathrm{s}$ are treated as classical vectors. In order to draw a phase diagram the authors introduced another control parameter:

$H_{3}=-E_{z} \sum_{i} \tau_{i}^{z}$

a 'magnetic field' for the orbital pseudo-spins, loosely associated with a uniaxial pressure along the $c$-axis. The classical limit phase diagram as function of $\eta$ and $E_{\mathrm{z}}$ is shown in Fig. 1.

For a detailed discussion of the various phases refer to [7]. To give some feeling, for large positive $E_{z}$ the $x^{2}-y^{2}$ orbitals are occupied, forming $(a, b)$ planes of anti-ferromagnetically coupled spins (AFxx). This is nothing else than the situation realized in, e.g. $\mathrm{La}_{2} \mathrm{CuO}_{4}$. For large negative $E_{z}$ the $3 z^{2}-r^{2}$ orbitals condense, forming a $3 \mathrm{D}$ spatially anisotropic Heisenberg antiferromagnet (Afzz with stronger exchange coupling along the $c$-axis than in the $(a, b)$ planes). Finally, the MOFFA, MOAFF and MOAAF phases are variations of the basic KK spin-orbital order [3] obtained by rotating the magnetic and orbital structure by $\pi / 2$. For the MoFFA phase at $E_{z}=0$, the orbitals have a twosublattice structure in the $(a, b)$-planes $\left(x^{2}-z^{2}\right.$ and $y^{2}-z^{2}$ on the A- and B-sublattice, respectively). Along the $c$-axis strong antiferromagnetic spin-spin couplings are found, while the spin couplings in the $(a, b)$ planes are ferromagnetic with a strength $\sim \eta$.

The anomaly occurs at the origin $\left(E_{z}, \eta\right)=(0,0)$ if the phase diagram: a 3D antiferromagnet (Afzz), a 2D antiferromagnet (Afzz) and a quasi-1A A-type antiferromagnet (MOFFA/MOAFF/MOAFF) become degenerate. The emphasis on the 'uniaxial pressure' $E_{z}$ is misleading in the sense that the full scope of the prob-

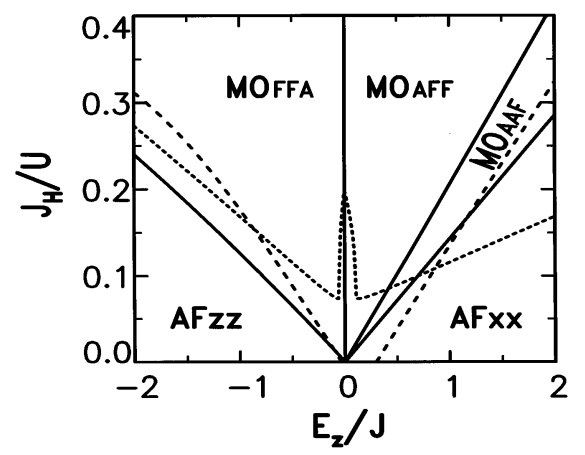

Fig. 1. Phase diagram of the KK model in the classical limit, as function of the Hund's rule coupling $J_{H}$ and tetragonal crystal field $E_{z}$ (reproduced from [7]). lem is not visible directly from this phase diagram: at the origin of Fig. 1 an infinity of classical phases become degenerate. This is trivial to understand. In the absence of Hund's rule exchange, the Hamiltonian Eq. (2) becomes the full story. Assuming a 3D classical antiferromagnet, $\vec{S}_{i} \cdot \vec{S}_{j}=-1 / 4$, and inserting this in Eq. (2) yields:

$H_{\mathrm{eff}}=J \sum_{\langle i j\rangle, \alpha}\left(\tau_{i}^{\alpha}+\tau_{j}^{\alpha}-1\right)$.

The orbital degrees of freedom are completely decoupled and all $2^{N}$ orbital configurations have the same energy $\left(\Sigma_{\alpha} \tau_{i}^{\alpha}=0\right)$. In addition, this infinity of different 3D spin systems has the same energy as the MOFFA/ MOAFF/MOAAF phases. It is actually so that at any finite temperature the $3 \mathrm{D}$ antiferromagnet becomes stable because of the entropy associated with the decoupled orbital sector [8].

This 'gauge' degeneracy is clearly a pathology of the classical limit. The authors continued by studying the stability of the classical limit, and by studying the stability of the classical phase diagram with respect to Gaussian quantum fluctuations. As discussed in more detail in [9] this is a somewhat subtle affair. Intuitively, one could be tempted to think that the orbitals and spins can be excited independently. This is however not the case. The dynamical algebra of relevance to the problem is an so(4) algebra, and this implies that modes will occur which excite at the same time the spins and the orbitals: the spin-and-orbital waves (SOW)s. Next to a (longitudinal) sector of pure orbital excitations, a 'transversal' sector is found corresponding with spin-excitations which are mixed with spill- and orbital-excitations, except for the acoustic modes at long wavelength which become pure spin-waves as imposed by the Goldstone theorem.

It was found that upon approaching the infinite critical point, the mass gap associated with the discrete symmetry in the orbital sector collapses. The (mixed) transverse modes give the dominating contribution to the renormalization of energy and magnetic order parameter. In the AFxx (AFzz) phase the lowest transverse mode softens along $\vec{k}=\left(\pi, 0, k_{z}\right)\left[\vec{k}=\left(k_{x}, 0,0\right)\right]$, and equivalent lines in the Brillouin zone (BZ), regardless how one approaches the critical lines. Thus, these modes become dispersionless along particular (softmode) lines in the BZ, where the finite masses were filled in the perpendicular directions,

$\omega_{\mathrm{AFxx}}(\vec{K}) \rightarrow \Delta_{x}+B_{x}\left(k_{x}^{4}+14 k_{x}^{2} k_{y}^{2}+k_{y}^{4}\right)^{1 / 2}$,

$\omega_{\mathrm{AFxx}}(\vec{K}) \rightarrow \Delta_{z}+B_{z}\left(k_{y}^{2}+4 k_{z}^{2}\right)$,

with $\Delta_{i}=0$ and $B_{i} \neq 0$ at the $M$ point, and the quantum fluctuations diverge logarithmically, $\left\langle\delta S^{z}\right\rangle \sim \int d^{3} k /$ $\omega(\vec{k}) \sim \int d^{2} k /\left(\Delta_{i}+B_{i} k^{2}\right) \sim \ln \Delta_{i}$, if $\Delta_{i} \rightarrow 0$ at the transition. It was found that the quantum correction to the 
order parameter $\left\langle S^{z}\right\rangle$ becomes large, well before the critical point is reached. In Fig. 1 the lines are indicated where $\|\left\langle\delta S^{z}\right\rangle \mid=\left\langle S^{z}\right\rangle$ : in the area enclosed by the dashed and clotted lines classical order cannot exist, at least not in gaussian order.

If the classical limit is as sick as explained in the previous paragraphs. what is happening instead? A priori it is not easy to give an answer to this question. There are no 'off' the shelf methods to treat quantum spin problems characterized by classical frustration, and the situation is similar to what is found in, e.g. $J_{1}-J_{2}-J_{3}$ problems [10]. A first possibility is quantum order-out-of-disorder [11]: quantum fluctuations can stabilize a particular classical state over other classically degenerate states. if this particular state is characterized by softer excitations than any of the other candidates. Khaliullin and Oudovenko [12] have suggested that this mechanism is operative in the present context, where the AFzz 3D anisotropic antiferromagnet is the one becoming stable. Their original argument was flawed because of the decoupling procedure they used, which violates the so(4) dynamical algebra constraints [9]. However, Khaliullin claims to found an 'so(4) preserving' self-consistent decoupling procedure which does yield order-out-of-disorder [13]. Nevertheless, there is yet another possibility valence-bond (VB) singlet (or spin-Peierls) order, which at the least appears in a more natural way in the present than is the case in higher dimensional spin-only problems, because it is favored by the directional nature of the orbitals.

The essence of a (resonating) valence bond [(R)VB] state is that one combines pairs of spins into Singlets. In the short-range (R)VB states these singlets involve nearest-neighbor spin pairs. Subsequently, one particular covering of the lattice with these 'spin-dimers might be favored (VB or spin-Peierls state), or the ground state might become a coherent superposition of many of these coverings (RVB state). On a cubic lattice the difficulty is that although much energy is gained in the formation of the singlet pairs. the bonds between the singlets are treated poorly Nevertheless, both in 1D Spin systems (Majumdar-Ghosh [14], AKLT-systems [15]) and in the large $N$ limit of $S U(N)$ magnets in 2D, ground states are found characterized by spin-Peierls/ VB order [16].

It is easy to understand that the interplay of orbitaland spin degrees of freedom tends to stabilize VB order. Since the orbital sector is governed by a discrete symmetry, the orbitals tend to condense in some classical orbital order. Different from the fully classical phases, one now looks for orbital configurations optimizing the energy of the spin VB configurations. The spin energy is optimized by having orbitals $3 \zeta^{2}-r^{2}$ on the nearest-neighbor sites where the VB spin-pair lives, with $\zeta$ directed along the bond. This choice maximizes the overlap between the wave functions, thereby the
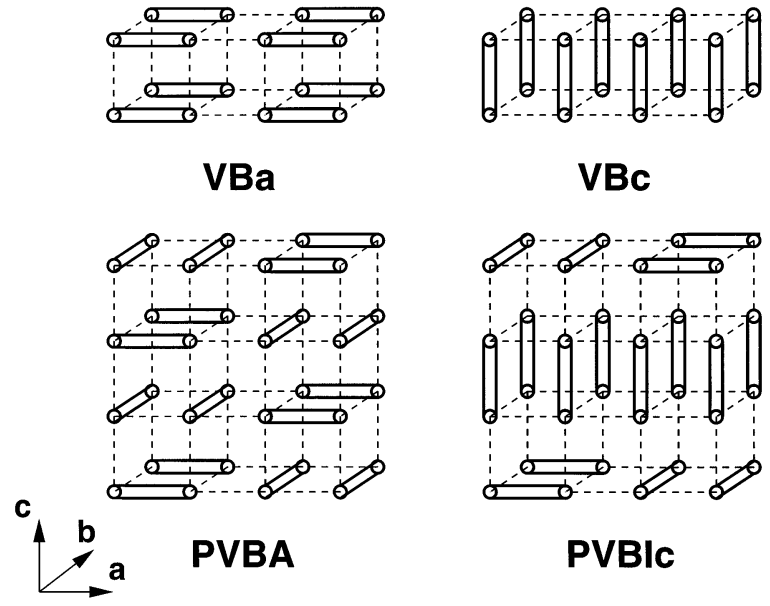

Fig. 2. A variety of valence bond solids (see text).

binding energy of the singlet. At the same time, this choice of orbitals minimizes the unfavorable overlaps with spin pairs located in directions orthogonal to $\zeta$. The net result is that VB states are much better variational solutions for the KK model, as compared to the standard Heisenberg spin systems.

Adressing this systematically, it was found that two families of VB states are most stable: (i) the 'staggered' VB states like the PVBA and PVBIc states of Fig. 2. These states have in common that the overlap between neighbouring VB pairs is minimized: the large lobes of the $3 \zeta^{2}-r^{2}$ wave functions of different pairs are never pointing to each other; (ii) the 'columnar' VB states like the $\mathrm{VBc}$ (or VBa) state of Fig. 2. In the orbital sector, this is nothing else than the AFzz state of Fig. 1 $\left(3 z^{2}-r^{2}\right.$ orbitals on every site). Different from the AFzz state, the spin system living on this orbital backbone is condensed in a 1D spin-Peierls state along the $z$-direction which is characterized by strong exchange couplings. The spins in the $a(b)$-directions stay uncorrelated, due to the weakness of the respective exchange couplings as compared to the VB mass gap.

The energies of these VB states and the classical states dressed up with quantum fluctuations are quite close together. A key issue is if the true ground state is compressible (dressed classical state), or characterized by a dynamical mass-gap (VB states). This will most likely depend on subtleties beyond the reach of the relatively crude variational Ansätze presented here ${ }^{1}$. So the nature of the ground state of the KK problem for small Hund's rule coupling is still an open problem.

\footnotetext{
${ }^{1}$ For instance one can argue that the columnar VB states are quite like the 'order-out-of-disorder' states of [12], with the only difference that the authors have imposed a spin-Peierls order in the chain direction.
} 


\section{Electron-phonon coupling in $\mathrm{KCuF}_{3}$}

In the previous section the orbital order as driven by the electron-electron interactions was discussed. However, one can think quite differently about the real systems: the deformations found in $\mathrm{KCuF}_{3}$ (or $\mathrm{LaMnO}_{3}$ ) could in principle be entirely caused by phonon-driven collective Jahn-Teller effects. This subject has been intensely studied in the past and is well understood. It starts out neglecting electron-electron interactions, and the focus is instead on the electronphonon coupling. In case that the ions are characterized by a Jahn-Teller (orbital) degeneracy, one can integrate out the (optical) phonons, and one finds effective Hamiltonians with phonon mediated interactions between the orbitals. In the specific case of $e_{g}$ degenerate ions in a cubic crystal, these look quite similar to the KK Hamiltonian, except that the spin dependent term is absent [17]. Any orbital order resulting from this Hamiltonian is now accompanied by a lattice distortion of the same symmetry.

The size of the quadrupolar deformation in the $(a, b)$ plane of $\mathrm{KCuF}_{3}$ is actually as large as $4 \%$ of the lattice constant $(a)$. It is therefore often argued that the orbital order is clearly phonon-driven, and that the physics of the previous section is an irrelevancy. Although appealing at first sight, this argument is flawed: large displacements do not necessarily imply that phonons do all the work.

The deformations of the lattice and the orbital degrees of freedom cannot be disentangled using general principles: they constitute an irreducible subsector of the problem. The issue is therefore a quantitative one, and in the absence of experimental guidance one would therefore like to address the issue with a quantitative electronic structure method. The LDA $+\mathrm{U}$ method is the method of choice. It is constructed to handle the physics of electronic orbital ordering, keeping the accurate treatment of the electron-lattice interaction of LDA intact. According to LDA + U calculations the total energy gained by the deformation of the lattice is minute as compared to the energies involved in the electronic orbital ordering [18]. At the same time, the phonons are important o the macroscopic scale and they contribute to driving $\mathrm{KCuF}_{3}$ away from the infinite-critical point of the phase diagram Fig. 1.

Based on the the initial observation that according to $\mathrm{LDA}, \mathrm{KCuF}_{3}$ would be an undistorted, cubic system: the energy increases if the distortion is switched on (Fig. 3). The reason is that $\mathrm{KCuF}_{3}$ would be a band metal according LDA (the usual Mott-gap problem) with a Fermi-surface which is not susceptible to a band Jahn-Teller instability. LDA $+\mathrm{U}$ yields a drastically different picture [18]. LDA can be looked at as unpolarized $\mathrm{LDA}+\mathrm{U}$, and by letting both the orbitals and the spins polarize an energy is gained of order of the band gap, i.e. of the order of $1 \mathrm{eV}$. The orbital and spin polarization is nearly complete and the situation is close to the strong coupling limit underlying the spin-orbital models of Section 2. Also when the cubic lattice is kept fixed, the correct orbital and spin ordering (MOFFA of Fig. 1) is found, with spin-exchange constants which compare favorably with experiment [18]. Because the orbital order has caused the electron density to become highly unsymmetric, the cubic lattice is unstable. Further energy can be gained by letting the lattice relax. The lattice distortion calculated in $\mathrm{LDA}+\mathrm{U}(\sim 3 \%$ of a) comes close to the actual distortion of $\mathrm{KCuF}_{3}(\sim$ $4 \%$ ). However, despite the fact that the distortion is large, the energy gained by the lattice relaxation is rather minute: $\sim 50 \mathrm{meV}$ (Fig. 3). Obviously, in the presence of the electronic orbital order the cubic lattice becomes very soft with regard to the quadrupolar distortions and even a small electron-phonon coupling can cause large distortions.

Although the energy gained in the deformation of the lattice is rather small, the electron-phonon coupling is quite effective in keeping $\mathrm{KCuF}_{3}$ away from the physics associated with the origin of the phase diagram (Fig. 1). Since the ferromagnetic interactions in the $(a, b)$ plane of $\mathrm{KCuF}_{3}$ are quite small $\left(J_{a b}=-0.2 \mathrm{meV}\right.$, as compared to the '1D' exchange $J_{c}=17.5 \mathrm{meV}$ [19]), one might argue that the effective Hund's rule coupling $J_{\eta}$ as of relevance to the low energy theory is quite small. Although this still needs further study, it might will be that in the absence of the electron-phonon coupling $\mathrm{KCuF}_{3}$ would be close to the origin of Fig. 1. However,

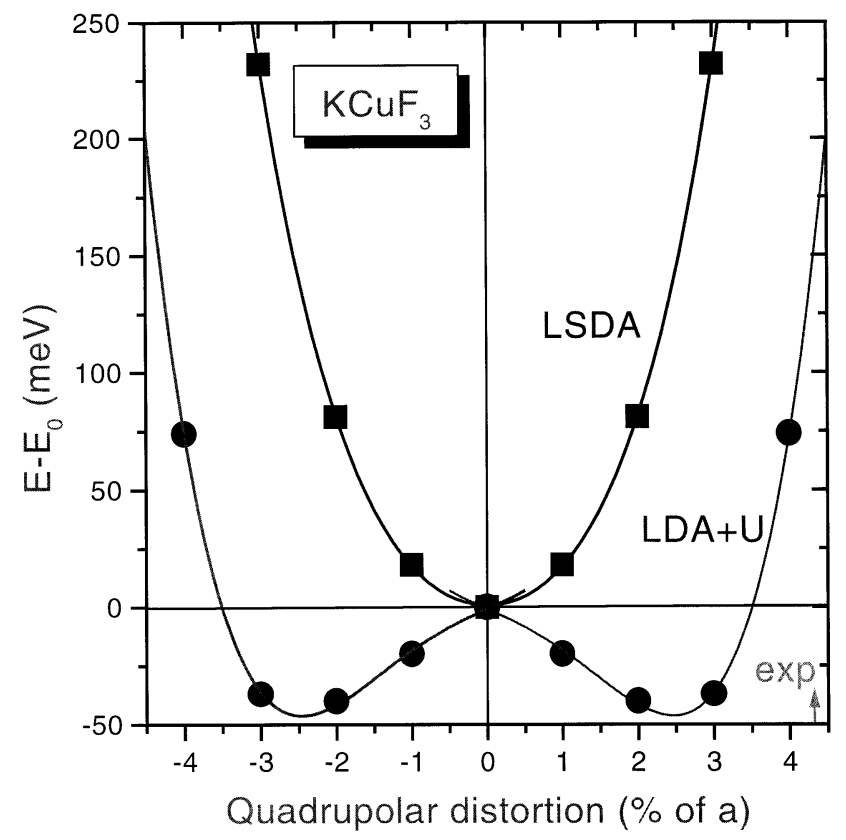

Fig. 3. The dependence of the total energy of $\mathrm{KCuF}_{3}$ on the quardrupolar lattice distortion according to LSDA and LDA $+\mathrm{U}$ band structure calculations (after [19]). 
the electron-phonon coupling introduces two scales: (i) a retardation scale, which is governed by the ratio of the phonon frequency and the electronic scale set by $J \sim 20 \mathrm{meV}$. Since $J$ is relatively small, $\mathrm{KCuF}_{3}$ is close to the anti-adiabatic limit where the lattice follows the electronic fluctuations; (ii) in the anti-adiabatic limit the phonons are high energy modes which can be integrated out, causing the effective orbital-orbital couplings earlier referred to. These couplings destroy the cancellations leading to Eq. (5), thereby driving the system away from the point of classical degeneracy. The typical scale for the phonon induced effective orbital interactions is at most of the order of the LDA + U lattice relaxation energy. However, as the latter $(\sim 50 \mathrm{meV})$ is quite a bit larger than $J$, the effective interaction will likely be able to put $\mathrm{KCuF}_{3}$, well outside the 'dangerous' region near the origin of the phase diagram.

In summary, although further work is needed it might be that phonons are to a large extent responsible for the stability of $\mathrm{KCuF}_{3}$ 's classical ground state. In any case, one cannot rely on the sheer size of the lattice deformations to resolve this issue.

\section{How about the manganites?}

Given the discussion so far, the search for interesting quantum effects in orbital degenerate Mott-insulators should not be regarded as hopeless. Unfortunately, the insulating parent compounds of the CMR manganities, such as $\mathrm{LaMnO}_{3}$, are not candidates for this kind of physics. The reason is not necessarily phonons; also in the manganites the 'Jahn-Teller' lattice distortions are sizeable, but this does not necessarily imply that the phonons are dominating. Two of us derived a KK-type model of relevance to this regime, and the authors did find a dynamical frustration of $e_{g}$-superexchange at $J_{H} \simeq 0$ [20]. However, the system is driven away from this point by two effects: (i) the manganites are in the Hund's rule dominated regime, with a large splitting between the lowest energy high-spin state at $U-5 J_{H}$ (with $J_{H}=0.69 \mathrm{eV}$ [21]), and the low-spin states at energies $\sim U$; (ii) the additional $t_{2 g}$-superexchange between the $S=3 / 2$ cores favours an antiferromagnetic order in all three spatial directions. The net outcome is that the ferromagnetic interaction between the total $S=2$ spins in the $(a, b)$ planes is of order of the $c$-axis exchange. signalling that the manganites are in the Hund's rule stabilized regime of the phase diagram.

The mysteries of the manganites relate to what happens when quantum-mechanical holes arc added to the orbital/spin ordered insulator. This is undoubt- edly a problem with its own characteristics, which cannot be reduced to a variation on the far simpler problems encountered in the insulators. Nevertheless, the authors believe that the study of the insulating limit might be of some help in better appreciating what is going on in the doped systems. It is tempting to think about orbital degrees of freedom as being spins in disguise. This is not quite the case. Orbitals are far less quantum-mechanical - they are more like Ising spins than Heisenberg spins. Secondly, orbitals carry this unfamiliar property that depending on their specific orientation in internal space, overlaps increase in particular real space directions, while they diminish in orthogonal directions. The valence-bond constructions illustrate this peculiar phenomen in the case of spins. but the same logic is at work when the hole is delocalizing. This intimate connection between internal symmetry and the directionality of delocalization causes the dynamical frustration which has been highlighted in this communication. This motive seems also at work in the doped system witness the many near degenerate states found both in mean-field calculations [21,22] and in experiment [23]. Further work is needed on this fascinating problem.

\section{Acknowledgements}

The authors thank A.I. Lichtenstein for helpful discussions. AMO acknowledges support by the Committee of Scientific Research (KBN) of Poland, Project No. 2 P03B 17514.

\section{References}

[1] For a review see: W. Bao et al., cond-mat/9804320.

[2] J. Zaanen, A.M. Olés, Phys. Rev. B 48 (1993) 7197.

[3] K.I. Krugel, D.I. Khomskii, Sov. Phys. Usp. 25 (1982) 231.

[4] L. Zaanen, A.M. Oleś, L.F. Feiner, in: G. Reiter et al. (Eds.), Dynamics of the Magnetic Fluctuations in High Temperature Superconductors, Plenum Press, New York, 1991, p. 241; L.F. Feiner, A.M. Oleś, J. Zaanen, J. Magn. Magn. Mat. 1941 (1995) $140-144$.

[5] A. Auerbach, Interacting Electrons and Quantum Magnetism, Springer, New York, 1994.

[6] D.P. Arovas, A. Auerbach, Phys. Rev. B 38 (1988) 316.

[7] L.F. Feiner, A.M. Olés, J. Zaanen, Phys. Rev. Lett. 78 (1997) 2799.

[8] J. Zaanen, unpublished.

[9] L.E. Feiner, A.M. Oleś, J. Zaanen, J. Phys. Condens. Matter 10 (1998) L555.

[10] P. Chandra, B. Doucot, Phys. Rev. B 38 (1988) 9335; A. Moreo et al., Phys. Rev. B 42 (1990) 6283. N. Read N. Sachdev, S. Sachdev, Phys. Rev. Lett. 66 (1991) 1773; A.V. Chubukov, D.K. Morr, Phys. Rev. B 52 (1995) 3521.

[11] A. Chubukov, Phys. Rev. B 44 (1991) 392.

[12] G. Khaliullin, V. Oudovenko, Phys. Rev. B 56 (1997) R14243. 
[13] G. Khaliullin, private communication.

[14] C.K. Majumdar, D.K. Ghosh, J. Math. Phys. 10 (1969) 1388.

[15] I. Affleck, T. Kennedy, E.H. Lieb, H. Tasaki, Phys. Rev. Lett. 77 (1996) 3G33.

[16] N. Read, S. Sachdev, Phys. Rev. Lett. 62 (1989) 1694.

[17] A.I. Liechtenstein, V.I. Khomskii, Sov. Phys. JETP 52 (1980) 501.

[18] A.I. Liechtenstein, V.I. Anisimov, J. Zaanen, Phys. Rev. B 52 (1995) R5467.
[19] D.A. Tennant et al., Phys. Rev. Lett. 70 (1993) 4003; Phys. Rev. B 52 (1995) 13368 and 13381.

[20] L.F. Feiner, A.M. Oleś, Phys. Rev. B 56 (1998) in press.

[21] T Mizokawa, A. Fujimori, Phys. Rev. B 51 (1995) 12880; Phys Rev. B 54 (1996) 0368.

[22] R. Maezono, S. Ishihara, N. Nagaosa, cond-mat/9805267.

[23] Y. Tokura et al., Phys. Soc. Jpn. 63 (1994) 3931; A. Urushibara et al., Phys. Rev. B 51 (1995) 14103; H. Kawano et al., Phys. Rev. Lett. 78 (1997) 4253. 